\title{
Clinical study on prevention of atopic dermatitis by oral administration of probiotics in infants
}

Jia-Huan He${ }^{1}$, Xin-Guo Zhao ${ }^{2}$, Feng Sun², Wen-Qi Peng ${ }^{2}$, Huan-Yun Li ${ }^{2}$ Hui Li ${ }^{2}$

'Department of Dermatology, First Affiliated Hospital of Dalian Medical University, Dalian, Liaoning, China

2Department of Taian, The 960 Hospital of the PLA, Taian, Shandong, China

Submitted: 17 June 2020; Accepted: 9 September 2020

Online publication: 5 November 2020

Arch Med Sci 2023; 19 (1): 101-106

DOI: https://doi.org/10.5114/aoms.2020.100639

Copyright @ 2020 Termedia \& Banach

\section{Abstract}

Introduction: This study aimed to investigate the preventive effects of oral administration of probiotics on the incidence and severity of atopic dermatitis (AD) in infants.

Material and methods: A total of 396 full-term infants were enrolled in this study. Of these, 132 newborns without a family history of AD were assigned to group $A$, and the other 264 newborns were randomly divided into groups $B$ and C. Infants in groups A and B were solely breastfed, while probiotics were administered to those in group $C$ as well as breastfeeding. The information of all subjects was recorded, and the incidence of $A D$ was followed up. The levels of serum IgE and IL-4 were measured at the age of 3 years.

Results: The incidence of $A D$ in infants in group $B$ was higher than that in group $A$ at 3 months, 4-6 months, and 7-36 months after birth, together with increased symptom scores. For infants in group $C$, the incidence of $A D$ at 4-6 months and 7-36 months after birth and the SCORAD scores at 0-3 months and 4-6 months after birth were lower than those in group B. The levels of IgE and IL-4 in group B were higher than those in groups A and $C$ at 36 months old.

Conclusions: Adding probiotics could favor the establishment of the intestinal microecological balance in the neonatal period, thereby reducing the incidence of $A D$, decreasing the levels of serum immune indexes and alleviating the severity of the disease.

Key words: atopic dermatitis, probiotics, neonatal period, SCORAD scores, breastfeeding.

\section{Introduction}

As one of the most concerning diseases in dermatology, atopic dermatitis (AD) is a common chronic, recurrent and inflammatory dermatosis that is often seen in children, and which is accompanied by a food allergy, allergic rhinitis, and allergic asthma. The patients have severe itching, which seriously affects their quality of life [1-3]. Approximately $45 \%$ of the patients developed the disease, which was characterized by pruritus, pleomorphism, and a tendency of exudation, within 6 months after birth. In the past 30 years, the incidence of AD has increased yearly, reaching $15-30 \%$ in children and $2-10 \%$ in adults $[4,5]$. Because the pathogenesis of $A D$ has not been fully elucidated, there are still difficulties in the treatment of AD. It has been suggested that vascular endothelial

\author{
Corresponding author: \\ Hui Li \\ Department of Taian \\ The 960 Hospital \\ of the PLA, \\ 271 Huanshan St \\ Taishan District \\ Taian 271000, China \\ Phone: 05388839479 \\ E-mail: lihui523600@163.com
}


growth factor (VEGF) and cell adhesion molecules correlate well with inflammation, favoring infiltration of the target tissue by mononuclear cells and thus causing the initiation and progression of the disease $[6,7]$. Free radicals such as $\mathrm{O}_{2}{ }^{-*}$, cytokines such as tumor necrosis factor $\alpha$ (TNF- $\alpha)$, adhesion molecules, dyslipidemia, and innate and adaptive immune responses may favor the vascular damage [8]. Thus, it is necessary to explore the pathogenesis and methods of prevention of AD.

The present study aimed to analyze the incidence of $A D$, together with the immune status of infants with $A D$ and a family history of atopic disease, by adding probiotics to food and exploring the preventive effect of oral probiotics on the incidence and severity of AD in infants.

\section{Material and methods}

\section{Study subjects}

In the present study, 396 full-term infants were admitted as the main subjects. Of these, 132 newborns without a family history were allocated to group A, and 264 newborns with a family history of atopic disease were randomly divided into groups B and C. Infants in group A and group B were solely breastfed, while probiotics were fed to infants in group $C$ in addition to breastfeeding. A family history of atopic disease refers to any member of the family who has AD, allergic asthma or allergic rhinitis, or other allergic diseases. The present study was in accordance with the Declaration of Helsinki of the World Medical Association and had been approved by the Ethics Committee of the hospital. The parents of all the infants signed their informed consent.

\section{Inclusion and exclusion criteria}

The inclusion criteria were as follows: 1) infants with 37 weeks $\leq$ gestational age $(\mathrm{GA})<42$ weeks and 2,500 g $\leq$ birth weight $<4,000 \mathrm{~g} ; 2$ ) infants without a history of intracranial hemorrhage, suffocation, rescue, infection, and congenital diseases, with the Apgar score $>7$ points; 3 ) no history of special medication, drug or alcohol abuse, or radiation exposure during pregnancy. Parents provided informed consent and accepted the breastfeeding plan without supplementary food for 6 months.

The exclusion criteria were as follows: 1) maternal complications during pregnancy; 2) small for gestational age; 3) infants being bottle-fed; and 4) infants whose mothers had chronic basic primary diseases such as diabetes, hyperthyroidism, and other endocrine diseases, chronic cardiopulmonary diseases, rheumatism, rheumatoid arthritis, chronic infectious diseases, or neurological and mental diseases.

\section{Methods}

All the newborns were breastfed. Infants in group A and group B were given no supplements, while infants in group $C$ received Clostridium caseinate live powder produced by Qingdao Donghai pharmaceutical (Clostridium butyricum powder, $500 \mathrm{mg}$ in each bag, $1.5 \times 10^{7} \mathrm{CFU} / \mathrm{g}$ of Clostridium butyricum in each bag) within 1-2 weeks after birth. The powder was administered a half bag at a time and twice daily, delivered with warm boiled water for ten days. The incidence of $A D$ was followed up, and the serum levels of IgE and IL-4 were measured when the children reached the age of three.

\section{Observation indexes and detection}

$A D$ could be diagnosed if three or more of the following five items were met: 1) history of skin involvement on the flexion side, including the elbow, popliteal fossa, anterior malleolus, or neck; 2) with a history or family history of other atopic diseases; 3) dryness of the skin; 4) existence of flexural eczema; and 5) with the onset of the disease before 2 years old. In the present study, since the participants were all infants with $A D$, the subjective symptom description was not accurate. Therefore, the SCORAD scoring method was used: i.e., $A / 5+7 B / 2$, where $A$ represents the area of skin lesions, with the scores ranging between 0 and 100 points; and B represents the severity of skin lesions, including six aspects: erythema, edema, desquamation, bryogenesis, exudation, and dryness, with 0-3 points allocated for each aspect, giving 0-18 points in total. Serum immunoglobulin $\mathrm{E}(\mathrm{IgE})$ and interleukin-4 (IL-4) were determined by the double-antibody sandwich ELISA. The unit of IgE was IU/ml, and that of IL-4 was $\mathrm{pg} / \mathrm{ml}$.

\section{Infants' information collection}

The children's electronic website was established, and the health files for all subjects were set. The probiotics treatment was completed within two to three weeks after birth. Physical examination was carried out 42 days, 3 months, 6 months, 9 months, 12 months, 18 months, 24 months, 30 months, and 36 months after birth. The infants were observed and followed up to collect data, so as to investigate the feeding condition, the existence of the disease, drug administration, and to guide treatment and nursing.

\section{Statistical analysis}

SPSS 20.0 software was used for statistical analysis. The measurement data were expressed as means \pm standard deviations $(\bar{x} \pm$ SD) and counting data in percentage (\%). The P-P chart method was used to test the normality, and Lev- 
Table I. Comparison of general conditions $(\bar{x} \pm$ SD)

\begin{tabular}{|c|c|c|c|c|c|c|c|c|}
\hline Variable & Male & Female & Complete & Fall off & $\begin{array}{l}\text { Gestational } \\
\text { age [week] }\end{array}$ & $\begin{array}{c}\text { Birth weight } \\
{[\mathrm{kg}]}\end{array}$ & $\begin{array}{l}\text { Body length } \\
{[\mathrm{cm}]}\end{array}$ & Type/effect \\
\hline Group A & 68 & 64 & 116 & 16 & $38.6 \pm 0.83$ & $3.43 \pm 0.37$ & $50.67 \pm 1.42$ & Normal \\
\hline Group B & 67 & 65 & 114 & 18 & $38.5 \pm 0.86$ & $3.42 \pm 0.39$ & $50.65 \pm 1.38$ & Normal \\
\hline Group C & 66 & 66 & 111 & 21 & $38.7 \pm 0.81$ & $3.44 \pm 0.45$ & $50.66 \pm 1.40$ & Normal \\
\hline Value ( $F$ or $\chi^{2}$ ) & \multicolumn{2}{|c|}{$0.061^{1}$} & $0.802^{1}$ & $1.618^{2}$ & $0.069^{2}$ & $0.006^{2}$ & $8 / 23$ & $\begin{array}{c}\text { Frameshift } \\
\text { variant }\end{array}$ \\
\hline$P$-value & \multicolumn{2}{|c|}{0.970} & 0.670 & 0.251 & 0.934 & 0.994 & $8 / 23$ & Normal \\
\hline
\end{tabular}

${ }^{1} \chi^{2}$ value; ${ }^{2}$ v value.

ene's test was used to test the homogeneity of variance. Univariate analysis of variance was used for multi-group comparison, and LSD was used for the post-test. A nonparametric test was used for comparison between groups that did not conform to the normal distribution. The counting data were expressed in frequency or rate, and the Pearson $\chi^{2}$ test was used to compare the differences between groups. $P$-value $<0.05$ was considered statistically significant.

\section{Results}

\section{General characteristics}

A total of 396 newborns who met the inclusion criteria were included in the present study. Of these, 132 newborns without a family history of $A D$ were allocated to group $A$, and 264 newborns with a family history of AD were randomly divided into groups $B$ and $C$. During the followup, 55 cases exited the study. The main reasons were the changes in residence, the poor compliance of family members, and the changes in feeding methods. There was no significant difference in the drop-out rate in each group. There was no significant difference in the living environment or the climate environment of the newborns and their parents. There was no significant difference in gender, birth weight, body length, age, and gestational age between groups $(p>0.05)$ (Table I).

\section{Incidence and severity of AD}

\section{Incidence and severity of AD at 3 months after birth}

The incidence of $A D$ in group $B$ was significantly higher than that in group $A$ at 3 months after birth, and the difference was statistically significant $(p<0.05)$. The SCORAD score at 3 months after birth in infants in group $B$ was significantly higher than that in group $A$, and the difference was statistically significant $(p<0.05)$. The SCORAD score at 3 months after birth in infants in group $C$ was significantly lower than that in group $B$, and the
Table II. Comparison of incidence of AD in $0-3$ months $(\bar{x} \pm \mathrm{SD})$

\begin{tabular}{|lccc|}
\hline Variable & $\begin{array}{c}\text { Total } \\
\text { number }\end{array}$ & $\begin{array}{c}\text { Incidence } \\
\text { of AD (\%) }\end{array}$ & $\begin{array}{c}\text { SCORAD } \\
\text { scores }\end{array}$ \\
\hline Group A & 116 & $24(20.68)$ & $12.72 \pm 2.21$ \\
\hline Group B & 114 & $41(35.96)^{\star}$ & $34.26 \pm 2.98^{*}$ \\
\hline Group C & 111 & $34(30.63)$ & $27.51 \pm 1.51^{\star \#}$ \\
\hline Value (F or $\left.\chi^{2}\right)$ & - & $6.715^{\text {a }}$ & $2600.494^{\text {b }}$ \\
\hline P-value & - & 0.035 & $<0.001$ \\
\hline
\end{tabular}

${ }^{a} \chi^{2}$ value, ${ }^{b} F$ value; pair-wise comparison, ${ }^{*}$ compared with group $A$, $p<0.05$; " compared with group $B, p<0.05$.

Table III. Comparison of incidence of AD in 3-6 months

\begin{tabular}{|lccc|}
\hline Variable & $\begin{array}{c}\text { Total } \\
\text { number }\end{array}$ & $\begin{array}{c}\text { Incidence } \\
\text { of AD (\%) }\end{array}$ & $\begin{array}{c}\text { SCORAD } \\
\text { scores }\end{array}$ \\
\hline Group A & 116 & $31(26.72)$ & $22.97 \pm 4.95$ \\
\hline Group B & 114 & $78(68.42)^{\star}$ & $45.06 \pm 5.16^{\star}$ \\
\hline Group C & 111 & $41(36.94)^{\#}$ & $26.91 \pm 5.01^{\star \#}$ \\
\hline Value (F or $\left.\chi^{2}\right)$ & - & $43.893^{\mathrm{a}}$ & $624.518^{\mathrm{b}}$ \\
\hline P-value & - & $<0.001$ & $<0.001$ \\
\hline
\end{tabular}

${ }^{a} \chi^{2}$ value, ${ }^{b} \mathrm{~F}$ value; pair-wise comparison, ${ }^{*}$ compared with group $A$, $p<0.05$; " compared with group $B, p<0.05$.

difference was statistically significant $(p<0.05)$ (Table II).

Incidence and severity of AD at 4-6 months after birth

As shown in Table III, the incidence of $A D$ in group $B$ was significantly higher than that in group $A$ at 4-6 months after birth, and the difference was statistically significant $(p<0.05)$. The symptom score at 4-6 months after birth in infants in group $B$ was significantly higher than that in group $A$, and the difference was statistically significant $(p<0.05)$. The incidence of $A D$ and the SCORAD score at 4-6 months after birth in infants 
Table IV. Comparison of incidence of AD in 6-36 months

\begin{tabular}{|lcc|}
\hline Group & Total number & Incidence of AD(\%) \\
\hline A & 116 & $2(1.72)$ \\
\hline B & 114 & $26(22.81)^{\star}$ \\
\hline C & 111 & $11(8.27)^{\star \#}$ \\
\hline
\end{tabular}

Fisher's precise test showed that $\chi^{2}=25.610, p<0.001$; pair-wise comparison, *compared with group $A, p<0.05$; " compared with group $B, p<0.05$.

Table V. Comparison of serum indicators at 36 months

\begin{tabular}{|lcc|}
\hline Variable & TIgE $[\mathrm{IU} / \mathrm{ml}]$ & $\mathrm{IL}-4[\mathrm{pg} / \mathrm{ml}]$ \\
\hline Group A & $15.18 \pm 8.15$ & $2.18 \pm 1.56$ \\
\hline Group B & $147.21 \pm 52.66^{*}$ & $9.11 \pm 2.97^{*}$ \\
\hline Group C & $85.19 \pm 40.25^{\star \#}$ & $5.09 \pm 2.12^{\star \#}$ \\
\hline F value & 339.834 & 265.460 \\
\hline P-value & $<0.001$ & $<0.001$ \\
\hline
\end{tabular}

F-test was used for uneven variance; pair-wise comparison, *compared with group A, $p<0.05$; ${ }^{*}$ compared with group $B, p<0.05$.

in group $C$ was significantly lower than those in group $B$, and the difference was statistically significant $(p<0.05)$.

Incidence and severity of AD at 7-36 months after birth

The incidence of $A D$ in group $B$ was significantly higher than in group $A$ at 7-36 months after birth, and the difference was statistically significant $(p<0.05)$. The incidence of $A D$ in group $C$ was significantly lower than that in group $B$ at 7-36 months after birth, and the difference was statistically significant $(p<0.05)$ (Table IV).

\section{Comparison of levels of IgE and IL-4 at 36 months old}

The levels of TIgE and IL-4 in infants in group $B$ were significantly higher than those in group $A$ at 36 months old, and the difference was statistically significant $(p<0.05)$. The levels of TIgE and IL-4 in infants in group $C$ were significantly lower than those in group B at 36 months old, and the difference was statistically significant $(p<0.05)$ (Table V).

\section{Discussion}

Many researchers have investigated $A D$ and believe that the underdevelopment of skin barrier, metabolism, and gastrointestinal function, together with the family history, conditions during pregnancy, postnatal lifestyle, and environment, were closely correlated with the occurrence of $A D$ [9-12]. The symbiotic microbial ecosystem formed by the intestinal flora is directly involved in human digestion, absorption, energy supply, immune regulation, fat metabolism, and many other aspects. It was previously reported in the literature that probiotics suppress the Th2 response and production of IL-4, IL-5, and IL-13 cytokines, and increase IL-10 and TGF- $\beta$ levels by inducing Treg cell function [13-15]. In the intestines, probiotics can stimulate the body to establish a perfect immune system. They then activate $T$ lymphocytes, promote the proliferation of $T$ lymphocytes, and play a positive role in the construction of the immune system in newborns [16-18].

At birth, the intestinal tract is sterile, and the immune system has not yet developed. During the process of growth, the intestinal flora begins to colonize and gradually reaches a stable state around the age of two. Under normal circumstances, the intestinal flora creates the immune response, putting the health of the host into a balanced state. The human body's microbial ecosystem is in dynamic balance with the health of the body, the surrounding environment, and diet throughout life. Studies [19-22] have shown that, in the early stage of life, if the exposure of microorganisms is reduced, the immune system of the body will be out of balance, leading to the occurrence of allergy-related atopic diseases. Thus, the "hygiene hypothesis" was proposed that early exposure to probiotics might promote the immune response, restore immune balance, and exert effects in the prevention and treatment of allergic atopic diseases [23-25].

The main atopic disease is generally anaphylaxis, in which the IgE antibody is the main factor of anaphylaxis, and IL-4 is the cytokine derived from $\mathrm{T}$ helper lymphocyte, which can promote the synthesis of IgE. IgE-related intestinal flora disorder in $A D$ was revealed through high throughput sequencing technology by foreign researchers [26]. In the present study, it was found that the incidence of $A D$ in infants with a family history was higher than in those without a family history, and the symptom score increased significantly in the former group, suggesting that the development of the disease was closely correlated with genetic factors. After taking prophylactic treatments, the incidence of $A D$ at 3-6 months and 24-36 months after birth, and the SCORAD scores at 0-3 months and 3-6 months in infants with a family history, were lower. These indicated that the application of probiotics in early life might reduce the incidence of disease. At 36 months, the levels of TIgE and IL-436 in group $B$ were higher than those in group $A$, while the levels decreased significantly in group $C$. Thus, it could 
be postulated that the addition of probiotics in the neonatal period might reduce the incidence and severity of $A D$ in infants with a family history, and the mechanism might be correlated with the improvement of serum immune indexes.

We found that the oral administration of probiotics could reduce the incidence of $A D$ in infants with a family history of atopic disease, as well as relieving the symptoms and significantly improving the levels of IgE and IL-4, which were regarded as important serum indexes of transient hypersensitivity. These observations suggest that probiotics could inhibit the high sensitivity state of the body and reduce the tendency of AD to occur, which means that probiotics might play a preventive role in the occurrence of other diseases such as allergic rhinitis and allergic asthma. The application of probiotics in early life, to intervene and improve the immune system of the newborn, is of great significance for the primary prevention of certain atopic diseases. The mechanism might be related to the regulation of the expression of inflammatory factors. Therefore, we recommend that in newborns with a family history of atopic diseases, the administration of appropriate probiotics might favor the formation of intestinal microecology and the establishment of microecology balance, and further improve the immune system.

There were some limitations to the present study. First, the sample size included in the present study was small, and it is necessary to increase the sample size and carry out multi-center clinical research. Second, the observation duration of the present study was short, and a long-term followup study is still needed.

In conclusion, adding probiotics could favor the establishment of the intestinal microecological balance in the neonatal period, thereby reducing the incidence of $A D$, decreasing the levels of serum immune indexes, and alleviating the severity of the disease.

\section{Acknowledgments}

Jia-huan He and Xin-guo Zhao are co-first authors.

We would like to acknowledge the hard and dedicated work of all the staff who implemented the intervention and evaluation components of the study.

\section{Conflict of interest}

The authors declare no conflict of interest.

\section{References}

1. Lucia B. Atopic dermatitis. Nat Rev Dis Primers 2018; 4: 2.

2. Dinakar C, Fineman SM, Tilles SA. Recent advances in atopic dermatitis. Ann Allergy Asthma Immunol 2018; 120: 8-9.
3. Jhamnani RD, Levin S, Rasooly M, et al. Impact of food allergy on the growth of children with moderate-severe atopic dermatitis. J Allergy Clin Immunol 2018; 141: 1526-9.

4. Gabes M, Chamlin SL, Lai JS, Cella D, Mancini AJ, Apfelbacher CJ. A study looking at changes in scores over time when using the childhood atopic dermatitis impact scale. Br J Dermatol 2020; 182: e67.

5. Ramadan M, Solyman S, Yones M, Abdallah Y, Halaby H, Hanora A. Skin microbiome differences in atopic dermatitis and healthy controls in Egyptian children and adults, and association with serum immunoglobulin E. OMICS 2019; 23: 247-60.

6. Murdaca G, Spanò F, Miglino M, Puppo F. Effects of TNF-alpha inhibitors upon the mechanisms of action of VEGF. Immunotherapy 2013; 5: 113-5.

7. Murdaca G, Colombo BM, Cagnati P, Gulli R, Spanò F, Puppo F. Endothelial dysfunction in rheumatic autoimmune diseases. Atherosclerosis 2012; 224: 309-17.

8. Murdaca G, Spanò F, Cagnati P, Puppo F. Free radicals and endothelial dysfunction: potential positive effects of TNF-alpha inhibitors. Redox Report 2013; 18: 95-9.

9. Huang T, Chen Y, Lin S, et al. Treatment of atopic dermatitis with dupilumab in Taiwan: dynamic changes of IgE levels as a potential response biomarker. Eur J Dermatol 2019; 29: 658-9.

10. Qin G, Huang J, Pan Y, et al. Topical ozone application: an innovative therapy for infantile atopic dermatitis. Zhong Nan Da Xue Xue Bao Yi Xue Ban 2018; 43: 163-7.

11. Brunner PM, Israel A, Zhang N, et al. Early-onset pediatric atopic dermatitis is characterized by $\mathrm{t} \mathrm{h} 2 / \mathrm{t} \mathrm{h} 17 / \mathrm{t} \mathrm{h}$ 22-centered inflammation and lipid alterations. J Allergy Clin Immunol 2018; 141: 2094-106.

12. Sorokman TV, Sokolnyk SV, Babiy OR, Lozyik IY. Immunological parameters and cortisol levels in children with atopic dermatitis. Arch Balkan Med Union 2018; 53: 210-6.

13. Marschan E, Kuitunen M, Kukkonen K, et al. Probiotics in infancy induce protective immune profiles that are characteristic for chronic low-grade inflammation. Clin Exp Allergy 2008; 38: 611-8.

14. Soh SE, Aw M, Gerez I, et al. Probiotic supplementation in the first 6 months of life in at risk Asian infants - effects on eczema and atopic sensitization at the age of 1 year. Clin Exp Allergy 2009; 39: 571-8.

15. Yao F, Jia R, Huang $H$, et al. Effect of Lactobacillus paracasei N1115 and fructooligosaccharides in nonalcoholic fatty liver disease. Hepatology 2019; 15: 1336-44.

16. Klinnert MD, Booster G, Copeland $M$, et al. Role of behavioral health in management of pediatric atopic dermatitis. Ann Allergy Asthma Immunol 2018; 120: 42-8.

17. Kim BE, Leung DYM. Significance of skin barrier dysfunction in atopic dermatitis. Allergy Asthma Immunol Res 2018; 10: 207-15.

18. Shinohara M, Matsumoto K. Atopic dermatitis and environmental factors associated with skin responses during infancy. J Allergy Clin Immunol 2019; 143: ab158.

19. Savilahti EM, Kukkonen AK, Haahtela T, Tuure T, Kuitunen $M$, Savilahti E. Intestinal defensin secretion in infancy is associated with the emergence of sensitization and atopic dermatitis. Clin Exp Allergy 2012; 42: 405-11.

20. Takahata Y, Sugita T, Kato H, Nishikawa A, Hiruma M, Muto M. Cutaneous malassezia flora in atopic dermatitis differs between adults and children. $\mathrm{Br} J$ Dermatol 2008; 157: 1178-82.

21. Bisgaard H, Li N, Bonnelykke K, et al. Reduced diversity of the intestinal microbiota during infancy is associated with increased risk of allergic disease at school age. J Allergy Clin Immunol 2011; 128: 646-52.e5. 
22. Sarkar S. Probiotic therapy for gastro-intestinal allergenic infants: a preliminary review. Br Food J 2007; 109: 481-92.

23. Flohr C, Pascoe D, Williams HC. Atopic dermatitis and the 'hygiene hypothesis': too clean to be true? Br J Dermatol 2005; 152: 202-16.

24. Enomoto T, Sowa $M$, Nishimori $K$, et al. Clinical effects of probiotic Bifidobacterium breve supplementation in adult patients with atopic dermatitis. Yonago Acta Medica 2010; 53: 37-45.

25. Prescott SL, Dunstan JA, Hale J, et al. Clinical effects of probiotics are associated with increased interferon-gamma responses in very young children with atopic dermatitis. Clin Exp Allergy 2006; 35: 1557-64.

26. Kasperkiewicz M, Schmidt E, Ludwig RJ, Zillikens D. Targeting IgE antibodies by immunoadsorption in atopic dermatitis. Front Immunol 2018; 9: 254. 